\title{
Fostering Social Resilience via Online Neighborhood Social Networks During the COVID-19 Pandemic and Beyond: Status Quo, Design Dilemmas and Research Opportunities
}

\author{
Pascal Vogel \\ University of Hamburg \\ pascal.vogel@uni-hamburg.de \\ Christian Grotherr \\ University of Hamburg \\ christian.grotherr@uni-hamburg.de
}

\author{
Christian Kurtz \\ University of Hamburg \\ christian.kurtz@uni-hamburg.de \\ Tilo Böhmann \\ University of Hamburg \\ tilo.boehmann@uni-hamburg.de
}

\begin{abstract}
Public health measures are curtailing the COVID-19 pandemic's spread but also impact individual and societal well-being. Altogether, they test the social resilience of communities, their collective ability to cope with crises. The pandemic highlights the significance of the immediate local community or neighborhood, be it for providing assistance to individuals in need, the sensible sharing of public spaces or a renewed conscience for supporting local businesses. We argue that online neighborhood social networks (ONSNs) represent a viable solution for improving social resilience as they enhance a community's resistance to disruptions, quicken recovery to a normal level of functioning and can become a platform for creative solutions to strengthening social resilience. We conduct a multiple case study to demonstrate how ONSNs foster social resilience in the focal crisis and beyond. Furthermore, we identify design dilemmas and highlight avenues for IS research with a high impact on local communities and their well-being.
\end{abstract}

\section{Introduction}

The COVID-19 outbreak has rapidly evolved from a regional epidemic to being designated a pandemic by the World Health Organization (WHO) [1]. At the time of writing, the WHO reports a total of six million laboratory-confirmed cases and more than half a million deaths caused by the coronavirus disease 2019 (COVID-19) [2]. Governments around the world are responding with public health measures such as physical distancing, mandatory quarantines, stay-athome orders and travel bans. While these measures show success in curtailing the spread of the COVID-
19 pandemic [3], they impact our individual and societal well-being. Potential adverse outcomes can range from boredom and frustration to stress and depression [4]. Many types of businesses that rely on physical proximity, are affected, cascading into severe long-term effects for entire economies [5]. Yet, a selection of public health measures will likely have to remain in place for an extended period of time until an effective vaccine for COVID-19 is widely available, at least for some regions or population groups [6].

Hence, the COVID-19 pandemic tests the collective ability of communities to respond positively to and cope with a crisis, their social resilience [7]. These responses highlight the significance of our immediate surroundings, our community or neighborhood, which during the COVID-19 pandemic play a key role in ensuring social resilience [8]. Vulnerable groups such as the elderly, children or the poor rely on assistance from local institutions, organizations and individuals to sustain themselves during this crisis. Physical distancing has necessitated new usage patterns in public transportation and shared usage of public spaces in general. Disruptions in the supply chains of some goods, as well as business models challenged by public health measures, have raised a newfound awareness for supporting local businesses [9]. But communities pulling together during this pandemic must do so intelligently and without risking infection, lest they worsen the very crisis they are trying to surmount.

At the same time, the diffusion of broadband internet and digital technology allows us to sustain at least some facets of our personal and professional lives, whether it is for maintaining social relationships, education, work or entertainment. Online social networks (OSNs), in particular, can play an important role in mitigating the effects of crises [10], including 
the current COVID-19 pandemic [11, 12]. Online neighborhood social networks (ONSNs) are a novel type of online social network (OSN) that affords their users functionality for social interaction and information sharing, peer support and integration of local service providers in a geographically delimited community of trustable neighbors [13]. These neighborhood platforms have gained in popularity over the last years, San-Francisco-based Nextdoor leading the charge with 27 million active monthly users and 236,000 active neighborhoods [14]. We argue that these capabilities make ONSNs a viable solution for fostering social resilience as they support a community's resistance to disruptions as well as recovery to a normal level of functioning. As they are already home to a like-minded community of neighbors and have reached a certain level of technological maturity and reliability, they are an instrument that is available for immediate application in the face of the current crisis. While there are first studies on the design and effects of ONSNs $[15,16]$ and they, among other OSNs, are finding application during the COVID-19 pandemic $[11,12,17]$, their role in improving social resilience has not yet been investigated. Furthermore, there is a need for further research investigating how citizens leverage social media for self-coordination and self-help in times of crisis [18]. Taking into consideration both the current crisis of the COVID-19 pandemic as well as future crises, we define the following guiding research question:

\section{How can online neighborhood social networks be leveraged to foster social resilience in local communities?}

With this research question, we respond to the current calls for information system (IS) research on building resilience to pandemics and other crises [19, 20]. To answer our research question, we conduct a multiple case study of ONSNs and analyze their general capabilities for fostering social resilience as well as the means by which they adapt their capabilities to the COVID-19 pandemic. We combine an in-depth analysis of user-generated content of one specific ONSN based in a German metropolitan area with a high-level analysis of capabilities of a multitude of ONSN platforms available globally. Based on these findings, we highlight the conflicting role of "local" in the face of the COVID-19 pandemic, how ONSNs can contribute to the intelligent orchestration of neighborhood resources and how IS research can contribute to fostering social resilience in case of the COVID-19 pandemic as well as future crises.

This paper is structured as follows. The next section provides an overview of previous research on
ONSNs and social resilience. The third section describes the applied case study research methodology, while the fourth section presents the results of our analysis. We discuss the implications of this study as well as opportunities for further research in the fifth section and conclude with a summary of its results, contributions and limitations.

\section{Related work}

\subsection{Online neighborhood social networks}

Neighborhood-centric information systems can be traced back to community computing initiatives such as the Blacksburg Electronic Village [21] or Netville [22], which provided neighbors with access to discussion boards, local news, email lists and business listings as early as the 1980s. With the rise of OSNs, cumulative and segmentative network effects led to the formation of neighborhood-focused subcommunities in the form of groups [23]. Online neighborhood social networks are a novel type of OSN whose functional and topical focus lies on neighborhood-related issues and that are aimed at the inhabitants of one or more spatially delimited neighborhoods [24]. Common functionality of ONSNs consists of a neighborhood-wide activity stream for sharing posts on topics such as local news, recommendations or requests for assistance in daily tasks, direct messaging between neighbors and neighbor profiles and directories [13]. While this set of features is similar to that of traditional OSNs such as Facebook, ONSNs differentiate themselves in a number of ways. A central design feature of ONSNs is the automated segmentation of users into isolated subcommunities based on their neighborhood of residence, determined via an address and identity verification mechanism ranging in rigorousness from sharing one's device location to in-person id checks. These mechanisms have been demonstrated to establish a community of trust amongst the members of some online neighborhoods $[13,25]$, increasing the value of ONSN content and features. For instance, trust in recommendations received, trades on a marketplace or willingness to request or provide assistance, which could technically also be found on traditional OSNs, could be of higher value in a community of trustful neighbors. Other frequently observed differences include a lack of user-to-user relationships such as friends lists or followers, a local offline footprint in form of representatives such as neighborhood managers and limited platform availability. Besides market leader Nextdoor, popular ONSNs include Berlin-based nebenan (German for 
next door) with 1.6 million users across several European countries [26] and Neighbourly with 830,000 users in New Zealand [27].

The term neighborhood can be defined from a multitude of perspectives [28] and ONSNs employ a variety of approaches to delineating the boundaries of neighborhoods. These range from crowdsourced delineation by users to radius-based approaches. While traditional OSNs such as Facebook aim to achieve maximum online engagement of users due to their business model [29], the goal construct of ONSNs is more ambiguous, positioning them more towards being facilitators of offline activity than online engagement. As a result, ONSN platform providers are required to develop novel business models that do not primarily rely on online advertising [13]. Studies analyzing the effects of ONSNs demonstrate their ability to increase neighborly communication and activities [16], to foster local engagement and intergenerational communication [15] and to improve social connectedness and participation among neighbors [30]. While ONSNs such as Nextdoor are also brought to bear alongside other OSNs during crises such as the COVID-19 pandemic [11], as yet, the focus in this regard has been generic communication and information sharing functionality as opposed to functionality targeted at improving social resilience.

\subsection{Technology-mediated social resilience}

Crises of natural and human origin as well as the accompanying disruption, challenge the resilience of communities. In this regard, social resilience describes "the capacity of social groups and communities to recover from, or respond positively to, crises" [7, p. 1]. Social resilience can be conceptualized based on the three properties of resistance, recovery and creativity $[7,31]$ (see Figure 1). Resistance describes the extent to which a community can withstand disruption without passing a point of no return, resulting in longterm negative consequences. Recovery can be understood as the time between a disruption and a community returning to its previous level of functioning. Finally, creativity describes how a community leverages experiences gained and adaptations made in the course of a disruption to achieve a permanently higher level of functioning compared to the pre-disruption base level. A community's social resilience is determined by a variety of attributes such as social and people-place networks, the knowledge and skills of its inhabitants, governance structures as well as infrastructure and economy [32].
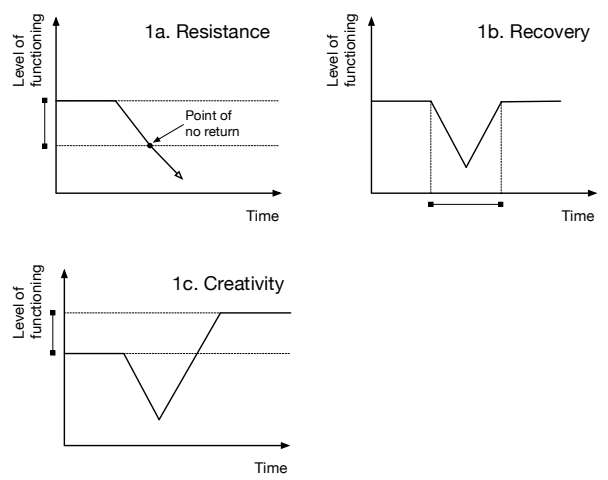

Figure 1. Properties of social resilience: resistance, recovery and creativity (adapted from Maguire and Hagan [7])

Digital technology can improve social resilience by facilitating access to education and health, enabling the monitoring of risks as well as by connecting and empowering communities. In particular, social media platforms can strengthen the social resilience of communities [11, 33, 34], for instance, by reducing risks for disaster through information distribution and education, assisting management and communication efforts during an active disaster or by developing communities of support amongst those affected by and responding to disasters and their aftereffects [35]. The cooperative use of social media by citizens and emergency services in times of crisis harbors the potential to foster social resilience, e.g., via emergent citizen groups and digital volunteering [33].

In case of the COVID-19 pandemic, social media is being leveraged to, among other capabilities, communicate health information, to build trustful relationships between citizens and public health institutions, to combat misinformation and to contribute to sense-making among the general population $[17,36]$. The nature, scale and severity of the COVID-19 pandemic have led to a call for further research on technology-mediated social resilience and crisis responses $[19,20]$. Cases, methods and tools for utilizing social media in communication between authorities and citizens have been extensively researched over the last decade [18]. However, there is a need for further research on the self-coordination and self-help practices employed by citizens on social media in times of crisis [18] and there is no research investigating the potential of ONSNs for supporting citizens in organizing self-help efforts.

\section{Research methodology}

We conduct a multiple case study [37] of ONSNs to investigate (1) how they foster social resilience 
during the COVID-19 pandemic and (2) how they are adapting their platform design features to face the challenges of the current crisis (see Figure 2). Our approach is T-shaped, performing both a deep analysis of user-generated data from one specific ONSN as well as a high-level analysis of platform design features from a broader selection of multiple additional ONSNs. We choose this approach because access to ONSNs is usually restricted to inhabitants of a specific neighborhood and this restriction is enforced via identity and address verification mechanisms, making it difficult to access user-generated content from an external perspective as opposed to publicfacing OSNs such as Twitter. The MyNeighbors ONSN platform, which is being operated by the authors as part of an ongoing design science research project in the context of healthy and connected communities [24, 30, 38], offers a unique opportunity to gain comprehensive access to user-generated content on an ONSN.

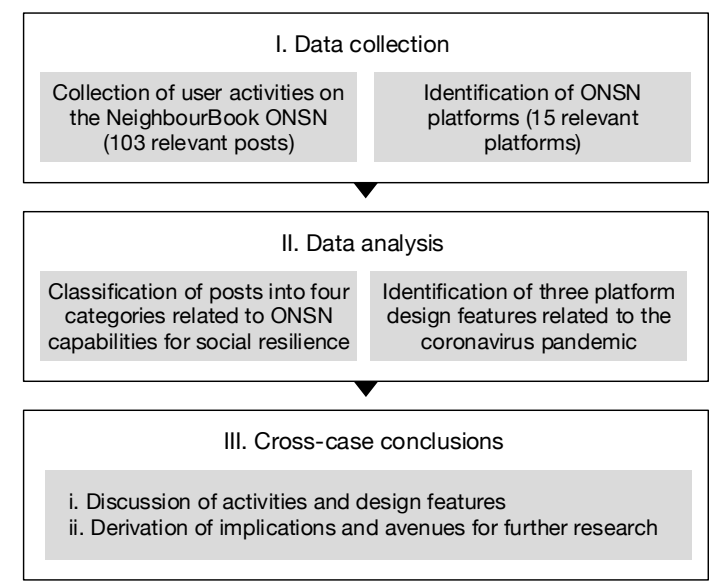

Figure 2. Research steps and activities

Choosing this approach, we are able to quickly develop first insights useful for coping with the COVID-19 pandemic while still drawing on a set of data that is both comprehensive and diverse. The final step of our research consists of the derivation of crosscase conclusions [37] by comparing the current state of usage and artifact design features of our set of ONSNs, determining commonalities and differences, and deriving implications for future research into leveraging ONSNs for fostering social resilience.

\subsection{Analysis of user-generated content}

To investigate how ONSNs foster social resilience during the COVID-19 pandemic, we collect user-generated content in the form of posts made to the
MyNeighbors ONSN. The platform is being used by around 160 users in two case neighborhoods in a large German metropolitan area and possesses the common set of ONSN features described in Section 2.1. In addition, MyNeighbors is integrated with a professional neighborhood management service that takes the role of offline and online community management as well as several local organizations and institutions such as health service providers, clubs, citizen's initiatives and churches. Between March $1^{\text {st }}$ and May $15^{\text {th }}, 2020,103$ out of a total of 128 posts made on MyNeighbors were related to the COVID-19 pandemic.

Three researchers independently analyzed the collected data. Our coding scheme was developed based on the core functional capabilities of ONSNs: information sharing, social interaction, peer support and integration with local organizations and institutions [30]. During data analysis, coders first identify those platform posts that are related to the current crisis of the COVID-19 pandemic. Subsequently, the remaining posts are deductively coded by each researcher using the developed coding scheme. Intercoder agreement was measured at $88.976 \%$ based on average pairwise percent agreement and .838 based on Krippendorff's Alpha. In a final harmonization step, the three coders discussed deviations in their code assignments until intercoder agreement was reached. The results of this coding procedure are presented in Section 3.1.

\subsection{Analysis of platform design features}

To investigate how ONSNs are adapting their platform design features to face the challenges of the current crisis, we collect and analyze data from publicly available ONSN platforms on their websites, knowledge bases and company blogs. To identify ONSN platforms, we perform a criteria-based online search using the company databases crunchbase (crunchbase.com) and CB Insights (cbinsights.com), the iOS App Store and Google Play Store as well as the Google search engine. We utilize combinations of the search terms neighborhood, community, local, social media and social network. We exclude platforms that have a neighborhood focus but do not fit our definition of ONSNs (e.g., security-focused platforms such as Neighbors by Ring) and local social networks without a neighborhood focus (e.g., local shopping apps such as Wiva). Based on these criteria, we identify and analyze fifteen ONSNs (see Appendix A). As platform access restrictions prevent the gathering of user-generated content in case of these platforms, we instead collect data on the "specific artifact capabilities" $[39$, p. 814] or design features of 
these platforms developed or adapted in response to the COVID-19 pandemic. We complement this data with third-party, public sources such as news articles or blog posts that report information on the response of these ONSNs to the COVID-19 pandemic. Of the fifteen platforms, two (nebenan and Nextdoor) have implemented novel design features in light of the COVID-19 pandemic.

\section{Results}

In this section, we present our results regarding both the usage of ONSNs based on data collected from the MyNeighbors ONSN (Section 4.1) and adaptations made to platform design features of ONSNs in response to the COVID-19 pandemic (Section 4.2).

\subsection{Community activity in response to the COVID-19 pandemic}

OSNs can represent a viable source of social connectedness [40], with ONSNs being no exception. ONSNs afford their users a variety of means for improving social connectedness and participation, including communication via posts or messages and by promoting social online and offline activities and offerings. Local communities, such as those using MyNeighbors, seek ways to stay connected and retain a sense of community belonging despite the interruption of in-person events. The collected data shows several instances of neighborhood-specific offerings for social interaction being made. A local church organized a photo contest for creative family activities compatible with physical-distancing, with winners being announced during online church service. Neighborhood managers arranged an outdoor exhibition of neighbor-provided photographs, while neighbors themselves arranged to regularly provide collective applause to essential workers at an agreedupon time every evening. The local church posted:

Who has the most creative ideas for good family time? Send us photos or descriptions of your common activities. [...] We want to inspire each other and will award the most original ideas in the next family church service!

Pandemic-induced public health measures such as physical distancing or stay-at-home orders have put a stop to many community activities, resulting in adverse outcomes such as social isolation and loneliness, particularly for vulnerable groups such as children or the elderly [41]. The analyzed data demonstrates how an ONSN supported the surveyed communities in maintaining existing social networks.
This, in turn, may counteract social isolation and loneliness and strengthen the overall crisis resistance and ability to recover from disasters.

The ONSN capability of sharing locally relevant information was used in connection with the COVID19 pandemic. Particularly at the pandemic's onset, information regarding public health measures on the national and state level, such as recommendations for personal hygiene or COVID-19 case numbers, was published. Furthermore, information regarding citylevel measures and their implementation in the local neighborhood were shared. The collected data, furthermore, shows instances of neighborhood-level announcements regarding topics such as policies regulating the use of public spaces and restrictions for neighborhood events being made. A neighborhood manager declared:

Dear members and neighbors, the assembly ban remains in place and the neighborhood common rooms continue to be unavailable for events. Any necessary exceptions [...] are only possible upon consultation and in accordance with appropriate safety measures.

Providing citizens with timely access to relevant information is an essential element of responding effectively to crises, building crisis resistance, the ability to recover and for fostering social resilience [18]. On the MyNeighbors ONSN, organizational representatives, neighborhood managers and neighbors made available locally relevant and targeted information related to the ongoing crisis. In case of MyNeighbors, neighborhood managers possess the critical role of not only distributing information but also monitoring information provided by other actors.

Establishing a local peer support network represents an important capability of ONSNs. By segmenting the inhabitants of neighborhoods into separate online communities, ONSNs offer neighbors a direct channel for requesting and provisioning volunteer support not automatically available on other OSNs. During the COVID-19 pandemic, a peer support network can provide vital assistance to at-risk individuals such as members of a group of the particularly vulnerable, ease the burden of confinement and prevent the violation of stay-at-home orders. The MyNeighbors ONSN received a number of posts related to the topic of peer support. These include unsolicited offers for assistance as well as public appeals to support each other made by individual neighbors or local organizations and institutions. For instance, two neighbors took the initiative and offered to provide shopping assistance to anyone in need. One neighbor stated: 
Dear neighbors, I am young, healthy, not part of a risk group, and have a lot of free time due to reduced working hours. If you need shopping assistance, feel free to contact me.

In case of the COVID-19 pandemic, an effective peer support network can immediately strengthen a community's resistance and ability to recover from crises, allowing at-risk individuals to safely isolate while being provided for. In the long term, it could also offer a path towards recovery to a near pre-crisis level of functioning with at-risk individuals remaining in isolation and in the care of members of their community while others are able to return to their daily lives. As ONSNs are oftentimes home to a community of like-minded and proximate individuals, they are an ideal platform for establishing such a network.

Local organizations and institutions published further offers for assistance, often in relation to their field of expertise. For example, a local shop offered emergency deliveries for groceries and neighborhood managers acted as contact points for referring individuals in need to available volunteers. The MyNeighbors ONSN affords organizational users with organizational profiles, a directory of local service offerings and a communication channel with neighbors via the platform. Organizations leveraged these design features as a means of communicating adaptations to existing and the launch of novel service offerings in response to the COVID-19 pandemic. This included updates on availability, i.e., cancellation or postponement, or mode of delivery, shifting from in-person to virtual approaches. For example, a local church brought attention to their new online church service and a yoga teacher presented video instructions. Organizations made use of the integrated neighborhood calendar to reflect these changes.

\subsection{ONSN adaptations in response to the COVID-19 pandemic}

Among the analyzed ONSNs, we identify three platform features adapted in response to the COVID19 pandemic. In March 2020, the ONSN platform nebenan received a novel design feature in the wake of the COVID-19 pandemic's increasing severity in Germany. The feature positions the ONSN as a matching platform between local volunteers and individuals in need as a result of the COVID-19 pandemic, strengthening its peer support capability. Requests for assistance are submitted by an individual in need via an automated phone hotline or online form without registering for the ONSN.

An interactive voice response system, in identical structure as the online form, collects a neighbor's name, postal code, contact information and type of required assistance. Proposed types include grocery shopping, household tasks or childcare. Alternatively, registered members can submit requests directly via the ONSN platform. Once submitted, requests for assistance are only visible to verified users in proximity to the requestor, i.e., in his or her neighborhood, which have undergone identity and address verification. Volunteer neighbors who accept a request for assistance are provided with the requestor's contact details and can mark a request as fulfilled upon completion.

Simultaneously, ONSN Nextdoor received a design feature called "Help Map" specifically targeted at mitigating the negative consequences of the COVID-19 pandemic. Using this feature, neighbors can announce their willingness to assist others by marking their location on an interactive map of their neighborhood and by specifying the types of assistance they are planning to provide. Individuals seeking assistance can use the same interactive map to identify helpful neighbors and contact them using Nextdoor's messaging system. Nextdoor describes grocery shopping or checking in on at-risk neighbors as potential services to be offered.

Both Nextdoor and nebenan aim to improve peer support but choose different approaches to achieve this goal. From the perspective of the individual in need, Nextdoor implements a "pull" approach where individuals in need pull a selection of available volunteers from the Help Map. This task needs to be actively repeated until a suitable volunteer is found. Nebenan implements a "push" approach, notifying individuals in need as soon as a volunteer accepts their published request for assistance. Nextdoor requires both requestors and volunteers to sign up for an account while this is the case only for volunteers on nebenan. For some user groups such as the elderly, it could be speculated that a phone call or online form may be more approachable than signing up for and using a web or mobile application, which entails an address verification. As opposed to nebenan, Nextdoor displays the locations of volunteers, providing individuals in need with an understanding of how close volunteers are located, which may positively influence individuals' inclination to accept help.

Besides peer support functionality, nebenan also adapted existing design features aimed at improving access to local service providers by offering a combination of marketing support, business stimulus and charitable fund-raising. Local businesses can sign up for a free business account on the platform, providing them with enhanced visibility, an additional communication channel and the opportunity to advertise products and services to a local audience. 
Neighbors can purchase gift cards for local businesses via nebenan, which can be redeemed after crisisrelated restrictions preventing a business from operating are lifted. With each purchased gift card, a donation of the same amount is made to the business by a pool of charitable partner organizations headed by nebenan. If a shop is not registered on nebenan, an employee will contact the owners and invite their participation in the initiative.

\section{Discussion}

In this section, we first discuss the implications of our results regarding the current state of using ONSNs for fostering social resilience (see Figure 3, [1]). Afterward, encountered dilemmas when designing for social resilience as well as future opportunities for IS research are highlighted (see Figure 3, [2]).

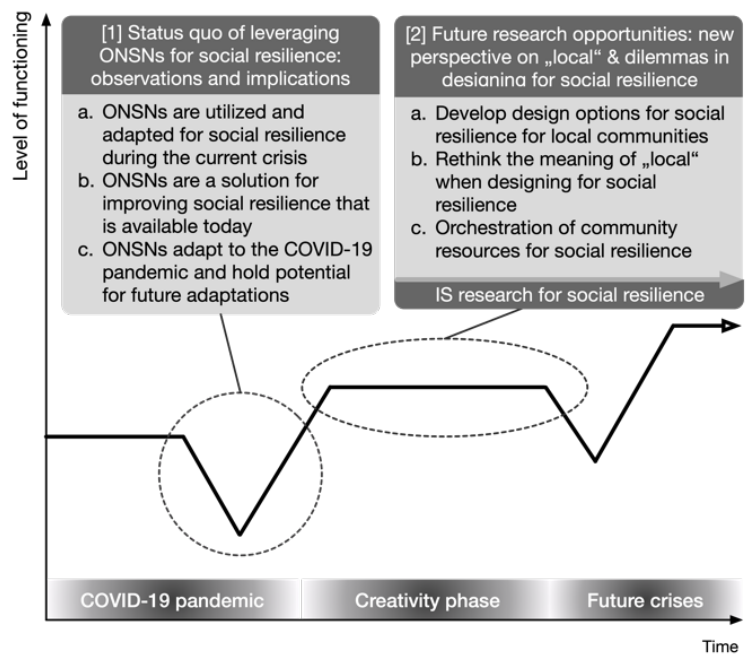

Figure 3. Overview of research results and implications for future IS research

\subsection{Leveraging ONSNs to strengthen the social resilience of local communities}

Our results show that ONSNs are being utilized to improve community resilience in the face of the COVID-19 pandemic, particularly by strengthening their resistance to and assisting their recovery from the crisis (see Figure 3, [1a]). They do so by maintaining social connections and a sense of belonging, via the distribution of locally relevant information, by establishing a peer support network and by improving access to local service providers. Strengthening the resilience of communities via ONSNs is not only valuable in case of the COVID-19 pandemic but can prove an investment in long-term preparedness for other crises and grand societal challenges such as population aging [42].

As ONSNs are readily available in many places and have reached a dependable technological and organizational maturity, they can provide immediate relief in the face of the COVID-19 pandemic. With the consequences of the pandemic expected to have a long-lasting impact on the well-being of communities and with a second wave of the pandemic as well as a repeated intensification of public health measures likely in many places [43], a timely establishment of measures for strengthening social resilience is imperative (see Figure 3, [1b]). As socio-technical artifacts, ONSNs can influence a community's practices and behaviors in the short term and shift norms, values and shared beliefs in the long term, ultimately contributing to the permanent institutionalization [44] of social resilience.

In light our results, we view ONSNs as a potential tool for improving social resilience by quickly adapting their design features to counteract negative consequences of the COVID-19 pandemic (see Figure 3 , [1c]). We observe novel platform features aimed at enhancing peer support capabilities as well as for supporting local businesses. Furthermore, neighbors and local organizations exhibited novel patterns of usage, recognizing and utilizing MyNeighbors as a suitable platform for meeting the challenges of the COVID-19 pandemic.

These observations highlight the unique role of technology, in this case ONSNs, as a dimension of the social resilience of communities. In contrast to inflexible dimensions such as a community's local infrastructure or economy [32], ONSNs can be made available quickly, possess high adaptivity to the requirements of a specific crisis and are a platform for generating creative novel solutions for social resilience. In sum, technologies represent touchpoints for engaged actors such as citizens, local service providers and governmental institutions. The development of suitable interventions is essential to integrate and embed ONSNs into the natural environment of these actors [45]. By linking sociotechnical artifacts designed to increase social resilience as well as empirically observable community behaviors on ONSNs, IS researchers can contribute evidence-based design knowledge for the last research mile in the triangle of rigor, relevance and design [46-49]. 


\subsection{A new perspective on "local" in light of the COVID-19 pandemic: dilemmas in designing for social resilience}

IS research is well-positioned to develop sociotechnical patterns of social resilience [19] in the context of ONSNs through the creative usage and analysis of existing platforms as well as by developing design knowledge through the design and evaluation of novel ONSN artifacts and capabilities. While still adjusting to the initial impact of the COVID-19 pandemic, we consider it of high importance to enter the creativity phase of social resilience [7] and to develop actionable insights as well as design options for meeting the immediate challenges of the current crisis and to empower our communities for those to come (see Figure 3, [2a]).

Resilient communities pull together in the face of a crisis, bringing to bear their local resources such as social networks, people-place connections and community infrastructure [32]. But in this regard, the COVID-19 pandemic poses a dilemma: physical proximity represents a risk and must, in many cases, be avoided to contain the pandemic's spread. Where previously a community's tight interconnectedness was an asset for resilience, it must now be scrutinized and carefully managed. Similar to individual efforts to not be deprived of social connections and a sense of belonging when distancing physically, communities should not be forced to sacrifice their social connectedness together with their local connectedness (see Figure 3, [2b]). But as digital communication is often still an imperfect substitute for in-person interaction, the solution cannot be an absolute shift from a place-based social resilience to a purely digital approach to social resilience. Any efforts aimed at improving community resilience in the face of the COVID-19 pandemic must acknowledge and incorporate these constraints.

Under these circumstances, ONSNs are wellpositioned to provide a form of hybrid social resilience, an intelligent orchestration of community resources that balances offline in-person interactions with online digital ones (see Figure 3, [2c]), for a number of reasons. Already, a pattern of ONSNs establishing themselves as platforms for peer support is evident in our results, with both Nextdoor and nebenan digitizing matchmaking and communication activities in order to enable the offline provisioning of volunteer services. But ONSNs have the potential to pursue this role even further, particularly in case of the COVID-19 pandemic. Their role as a platform interconnecting local actors and resources could be leveraged for the safe utilization of local shared spaces, e.g., community gathering places, parks or playgrounds, via mechanics such as booking or capacity management systems as well as by keeping a record of usage for contact tracing. This could serve to improve the resistance of communities by protecting at-risk individuals while at the same time, others can recover to a normal level of functioning.

Governments aim to improve the well-being of city inhabitants via digital technology [50], particularly in response to the COVID-19 pandemic [12]. These efforts should be expanded to not only aim at improving community well-being but also social resilience. Partnerships with public and private actors, e.g., providers in the housing, long-term care or technology sectors but also home delivery services, could complement volunteer-based peer support networks on ONSNs with professional service providers where necessary, more efficient or legally required.

\section{Conclusion}

In this study, we conduct a multiple case study of ONSNs and analyze their general capabilities for fostering social resilience as well as the means by which they adapt their capabilities to the COVID-19 pandemic. Our study has two main contributions. First, it demonstrates how ONSNs can support community resistance and recovery and, in turn, improve the social resilience of local communities during a crisis such as the COVID-19 pandemic. Furthermore, ONSNs are presented as a uniquely adaptive and flexible resource for fostering social resilience which can have a lasting positive effect on the level of functioning of a community beyond times of crisis. In the short-term, these results can offer guidance to local communities in coping with the coming months and years.

Second, we present future research opportunities in the context of ONSNs that build on the dilemma of "local" during the COVID-19 pandemic and leverage the unique position of ONSNs as intelligent orchestrators of community resources. We call for further IS research targeted at improving the long-term social resilience as well as disaster preparedness of local communities in order to develop creative solutions to meet the diverse challenges and adverse consequences arising in the course of crises such as the COVID-19 pandemic.

This research is subject to limitations. Our selection of ONSNs for analysis is biased as it is intentionally limited to the MyNeighbors ONSN as well as a set of English and German language 
platforms discovered via an internet search. We exclusively analyze user-generated content from the MyNeighbors ONSN, resulting in insights with limited generalisability due to the platform's limited availability and the small amount of data. However, we gain timely and comprehensive access and remain situated in the research context of our ongoing design science research project. We focus our comparison of other ONSNs on their externally visible platform design features. An in-depth analysis of usergenerated content in cooperation with other ONSN platforms could yield additional insights regarding their contribution to social resilience, particularly during the COVID-19 pandemic.

\section{Acknowledgments}

This research was funded by the European Regional Development Fund and the City of Hamburg as part of the research project AGQua (https://www.agqua.de).

\section{References}

[1] WHO. 2020. WHO Director-General's opening remarks at the media briefing on COVID-19 - 11 March 2020.202004-28, https://tinyurl.com/vyvm6ob

[2] WHO. 2020. Situation Report COVID-19 - 175 (July 13, 2020). 2020-06-01, https://tinyurl.com/y3ejt3nd

[3] Cowling, B.J., Ali, S.T., Ng, T.W.Y., Tsang, T.K., Li, J.C.M., Fong, M.W., Liao, Q., Kwan, M.Y.W., Lee, S.L., Chiu, S.S., Wu, J.T., Wu, P., and Leung, G.M. Impact assessment of non-pharmaceutical interventions against coronavirus disease 2019 and influenza in Hong Kong: an observational study. The Lancet Public Health, 5, 5 (2020), e279-e288.

[4] Pfefferbaum, B., and North, C.S. Mental Health and the Covid-19 Pandemic. New England Journal of Medicine (2020), 1-3.

[5] McKee, M., and Stuckler, D. If the world fails to protect the economy, COVID-19 will damage health not just now but also in the future. Nature Medicine, 26, 5 (2020), 640-642.

[6] Cohen, J. Vaccine designers take first shots at COVID19. Science, 368, 6486 (2020), 14.

[7] Maguire, B., and Hagan, P. Disasters and Communities: Understanding Social Resilience. Australian Journal of Emergency Management, 22, 2 (2007), 16-20.

[8] Beardmore, A., Jones, M., Biddle, M., White, J., Ismail, S., McClean, S., Gibson, A., and Sabey, A. Apart but not alone - Neighbour support and the Covid-19 lockdown. Bristol, UK: University of Bristol, 2020.

[9] Roggeveen, A.L., and Sethuraman, R. How the COVID Pandemic May Change the World of Retailing. Journal of Retailing (2020).

[10] Eismann, K., Posegga, O., and Fischbach, K. Collective Behaviour, Social Media, and Disasters: A Systematic Literature Review. European Conference on Information Systems, Istanbul, Turkey, 2016.
[11] Kummitha, R.K.R. Smart technologies for fighting pandemics: The techno- and human-driven approaches in controlling the virus transmission. Government Information Quarterly (2020), 101481.

[12] United Nations. 2020. COVID-19: Embracing digital government during the pandemic and beyond. 2020-06-03, https://tinyurl.com/y6yltdao

[13] Vogel, P., Grotherr, C., Kurtz, C., and Böhmann, T. Conceptualizing Design Parameters of Online Neighborhood Social Networks. International Conference on Wirtschaftsinformatik, Potsdam, Germany, 2020.

[14] Roof, K. 2019. Nextdoor Valued at More Than \$2 Billion in Fresh Funding Round. 2020-03-18, https://tinyurl.com/yysu29mc

[15] Renyi, M., Gündogdu, R., Kunze, C., Gaugisch, P., and Teuteberg, F. The Networked Neighborhood. IEEE International Conference on Engineering, Technology and Innovation, Konstanz, Germany, 2018.

[16] Masden, C.A., Grevet, C., Grinter, R.E., Gilbert, E., and Edwards, W.K. Tensions in Scaling-Up Community Social Media: A Multi-Neighborhood Study of Nextdoor. International Conference on Human Factors in Computing Systems, Toronto, Canada, 2014.

[17] Mirbabaie, M., Bunker, D., Stieglitz, S., Marx, J., and Ehnis, C. Social media in times of crisis: Learning from Hurricane Harvey for the coronavirus disease 2019 pandemic response. Journal of Information Technology, 19.

[18] Reuter, C., and Kaufhold, M.-A. Fifteen years of social media in emergencies: A retrospective review and future directions for crisis Informatics. Journal of Contingencies and Crisis Management, 26, 1 (2018), 41-57.

[19] Rai, A. Editor's Comments: The COVID-19 Pandemic: Building Resilience with IS Research. Management Information Systems Quarterly, 44, 2 (2020), iii-vii.

[20] Ågerfalk, P.J., Conboy, K., and Myers, M.D. Information systems in the age of pandemics: COVID-19 and beyond. European Journal of Information Systems (2020), 1-5.

[21] Carroll, J.M., and Rosson, M.B. Developing the Blacksburg electronic village. Communications of the ACM, 39, 12 (1996), 69-74.

[22] Hampton, K., and Wellman, B. Neighboring in Netville: How the Internet Supports Community and Social Capital in a Wired Suburb. City \& Community, 2, 4 (2003), 277-311. [23] Ilena, M., Ard, H., and Wim, B. On the Development of Online Cities and Neighborhoods: an Exploration of Cumulative and Segmentive Network Effects in Social Media. European Conference on Information Systems, Barcelona, Spain, 2011.

[24] Vogel, P., Grotherr, C., and Böhmann, T. Designing Tool Support for Crowd-Sourced Community Initiatives on Online Neighborhood Social Networks. European Conference on Information Systems, Marrakech, Morocco, 2020.

[25] De Meulenaere, J., Baccarne, B., Courtois, C., and Ponnet, K. Neighborhood hotspot and community awareness: The double role of social network sites in local communities. Communications, 0 (2020), 000010151520190135. 
[26] nebenan. 2020. Zahlen und Fakten zu nebenan.de. 2020-05-29, https://tinyurl.com/yxt7qgsu

[27] Neighbourly. 2020. Neighbourly shines in a time of coronavirus. 2020-05-23, https://tinyurl.com/y6zq3nry

[28] Sampson, R.J., Raudenbush, S.W., and Earls, F. Neighborhoods and Violent Crime: A Multilevel Study of Collective Efficacy. Science, 277, 5328 (1997).

[29] Montag, C., Lachmann, B., Herrlich, M., and Zweig, K. Addictive Features of Social Media/Messenger Platforms and Freemium Games against the Background of Psychological and Economic Theories. Int. Journal of Environmental Research and Public Health, 16, 14 (2019), 2612.

[30] Vogel, P., Jurcevic, N., and Meyer-Blankart, C. Healthy, Active and Connected: Towards Designing an AgeFriendly Digital Neighborhood Platform. European Conference on Information Systems, Stockholm-Uppsala, Sweden, 2019.

[31] Kimhi, S., and Shamai, M. Community resilience and the impact of stress: Adult response to Israel's withdrawal from Lebanon. Journal of Community Psychology, 32, 4 (2004), 439-451.

[32] Maclean, K., Cuthill, M., and Ross, H. Six attributes of social resilience. Journal of Environmental Planning and Management, 57, 1 (2014), 144-156.

[33] Reuter, C., and Spielhofer, T. Towards social resilience: A quantitative and qualitative survey on citizens' perception of social media in emergencies in Europe. Technological Forecasting and Social Change, 121 (2017), 168-180.

[34] Belblidia, M.S. Building Community Resilience Through Social Networking Sites: Using Online Social Networks for Emergency Management. International Journal of Information Systems for Crisis Response and Management, 2, 1 (2010), 24-36.

[35] Dufty, N. Using social media to build community disaster resilience. Australian Journal of Emergency Management, 27, 1 (2012), 40-45.

[36] Chen, Q., Min, C., Zhang, W., Wang, G., Ma, X., and Evans, R. Unpacking the black box: How to promote citizen engagement through government social media during the COVID-19 crisis. Computers in Human Behavior, 110 (2020), 106380.

[37] Yin, R.K., and Campbell, D.T. Case Study Research and Applications: Design and Methods. Thousand Oaks, California: SAGE, 2018.

[38] Grotherr, C., Vogel, P., and Semmann, M. Multilevel Design for Smart Communities: The Case of Building a Local Online Neighborhood Social Community. Hawaii International Conference on System Sciences, Grand Wailea, HI, USA, 2020.

[39] Meth, H., Mueller, B., and Maedche, A. Designing a Requirement Mining System. Journal of the Association for Information Systems, 16, 9 (2015), 799-837.

[40] Grieve, R., Indian, M., Witteveen, K., Anne Tolan, G., and Marrington, J. Face-to-face or Facebook: Can social connectedness be derived online? Computers in Human Behavior, 29, 3 (2013), 604-609.

[41] Brooke, J., and Jackson, D. Older people and COVID19: Isolation, risk and ageism. Journal of Clinical Nursing, 29, 13-14 (2020), 2044-2046.
[42] Hoffman, G.J., Webster, N.J., and Bynum, J.P.W. A Framework for Aging-Friendly Services and Supports in the Age of COVID-19. Journal of Aging \& Social Policy (2020), $1-10$.

[43] Xu, S., and Li, Y. Beware of the second wave of COVID-19. The Lancet, 395, 10233 (2020), 1321-1322.

[44] Lawrence, T.B., Leca, B., and Zilber, T.B. Institutional Work: Current Research, New Directions and Overlooked Issues. Organization Studies, 34, 8 (2013), 1023-1033.

[45] Benoit, S., Klose, S., Wirtz, J., Andreasen, T.W., and Keiningham, T.L. Bridging the Data-Divide Between Practitioners and Academics: Approaches to Collaborating Better to Leverage Each Other's Resources. Journal of Service Management, 30, 5 (2019), 524-548.

[46] Hevner, A.R. A three cycle view of design science research. Scandinavian Journal of Information Systems, 19, 2 (2007), 87-92.

[47] Cole, R., Purao, S., Rossi, M., and Sein, M. Being Proactive: Where Action Research Meets Design Research. International Conference on Information Systems, Las Vegas, USA, 2005.

[48] Nunamaker, J.F., Briggs, R.O., Derrick, D.C., and Schwabe, G. The Last Research Mile: Achieving Both Rigor and Relevance in Information Systems Research. Journal of Management Information Systems, 32, 3 (2015), 10-47.

[49] Iivari, J. Distinguishing and contrasting two strategies for design science research. European Journal of Information Systems, 24, 1 (2015), 107-115.

[50] Marrone, M., and Hammerle, M. Smart Cities: A Review and Analysis of Stakeholders' Literature. Business \& Information Systems Engineering, 60, 3 (2018), 197-213.

\section{Appendix}

\section{Appendix A. List of analyzed ONSN platforms}

\begin{tabular}{ll}
\hline \multicolumn{1}{c}{ Name } & \multicolumn{1}{c}{ URL } \\
\hline fürenand.ch & https://fuerenand.ch/ \\
FragNebenan & https://fragnebenan.com/ \\
GoNeighbour.org & https://www.goneighbour.org/ \\
JustMyNeighbors & https://justmyneighbors.com/ \\
lokalportal & https://lokalportal.de/ \\
Kiekmo & https://kiekmo.hamburg/ \\
Meine Nachbarn & https://meinenachbarn.hamburg \\
Meet the Neighbors & http://meettheneighbors.org/ \\
Nachbarschaft.net & https://nachbarschaft.net/ \\
nebenan & https://nebenan.de/ \\
Neighbourly & https://www.neighbourly.co.nz/ \\
Nextdoor & https://nextdoor.com// \\
Scooploop & https://www.scooploop.com/ \\
Remishueb & https://remishueb.stadt.sg.ch/ \\
wirRauner & https://www.wir-rauner.de/ \\
\hline
\end{tabular}

